\title{
Testosterona falsamente elevada em uma paciente diabética tipo 1 com acne e insuficiência renal crônica em diálise
}

\author{
Falsely elevated testosterone in a type 1 diabetes patients \\ with acne and chronic renal failure on dyalisis
}

José Antonio Miguel Marcondes' ${ }^{1}$ Thais Cotrim Martins', Alexandre Sarubbi Raposo do Amaral' ', Márcia Nery'

\section{SUMÁRIO}

Descrevemos uma paciente com diabetes tipo $1 \mathrm{com}$ acne e insuficiência renal crônica, em diálise, que apresentou uma dosagem de testosterona total e livre elevada $(612 \mathrm{ng} / \mathrm{dL}$, normal < 90 ng/dL e 255 pMol/L, normal: 20-45 pMol/L, respectivamente). Na avaliação clínica, além da acne facial não havia qualquer outro sinal de hiperandrogenismo. Após ter esse resultado confirmado, ela foi submetida à avaliação morfológica de adrenal e ovários (tomografia computadorizada de adrenal e ultrassom pélvico), cujos resultados foram normais. Na ausência de quadro clínico de virilização, foram consideradas outras possibilidades que pudessem explicar a elevação da testosterona, entre as quais a presença de comorbidades (diabetes e insuficiência renal crônica) e falha do método de dosagem. Uma nova determinação da testosterona total, por meio da cromatografia líquida de alta performance como método preparativo e espectrometria de massa em tandem, resultou normal $(21 \mathrm{ng} / \mathrm{dL})$, compatível com uma concentração de testosterona total falsamente elevada pela presença de interferentes com o método de dosagem. Arq Bras Endocrinol Metab. 2012;56(5):319-23

\section{SUMMARY}

The purpose of this report is to present the case of a patient with type 1 diabetes with acne and chronic renal failure on dialysis admitted to the hospital with high total total and free testosterone (612 ng/dL, normal < $90 \mathrm{ng} / \mathrm{dL} ; 255 \mathrm{pMol} / \mathrm{L}$, normal: 20-45 pMol/L). On clinical evaluation, she presented facial acne, and no other signs of hyperandrogenism. As this result was confirmed, she underwent adrenal and ovary morphological assessment (adrenal CT and pelvic ultrasound), which yielded normal results. Due to divergence between clinical and laboratory findings, we considered other possibilities that could explain the elevation of testosterone, including the presence of comorbidities (diabetes and chronic renal failure) and failure of the testosterone assay. Testosterone levels were determined again by high performance liquid chromatography, as a preparative method, and tandem mass spectrometry, yielding normal results $(21 \mathrm{ng} / \mathrm{dL})$, which were compatible with a falsely elevated total testosterone level caused by the presence of factors that intereferred with the assay. Arq Bras Endocrinol Metab. 2012;56(5):319-23
${ }^{1}$ Serviço de Endocrinologia do Hospital das Clínicas de São Paulo, São Paulo, SP, Brasil

\section{INTRODUÇÃO}

s síndromes hiperandrogênicas englobam doenças
ológica dos manifestam pelo aumento da atividade bi-
ções incluem hirsutismo, acne, alopecia tipo androgêni-
ca, disfunção menstrual, infertilidade, abortamento
precoce e sinais de virilização (atrofia do parênquima mamário, alteração da tonalidade da voz, redistribuição de massas musculares e clitoromegalia).

Tanto a testosterona total (forma livre e forma ligada às proteínas transportadoras) quanto a testosterona livre são consideradas marcadores sensíveis para a demonstração da hiperandrogenemia (1). Entretanto, não existe correlação entre a concentração dos andrógenos 
circulantes e o grau de hirsutismo, mas sim entre a taxa de produção de testosterona e as manifestações clínicas (2), permitindo a subdivisão das síndromes hiperandrogênicas em dois grupos: síndromes virilizantes, caracterizadas pela presença de sinais de virilização (neoplasias ovarianas e adrenais e hiperplasia do estroma cortical) e síndromes não virilizantes (forma não clássica de hiperplasia adrenal congênita, hirsutismo idiopático e síndrome dos ovários policísticos).

As síndromes virilizantes se caracterizam do ponto de vista fisiopatológico por uma taxa de produção elevada de testosterona e, clinicamente, pela presença dos sinais de virilização. Laboratorialmente, $80 \%$ das pacientes têm uma concentração sérica de testosterona total elevada, maior que $200 \mathrm{ng} / \mathrm{dL}$, quando dosada por radioimunoensaio. As síndromes hiperandrogênicas não virilizantes se caracterizam por apresentar uma menor taxa de produção de testosterona, pela ausência de sinais de virilização e por uma concentração de testosterona normal ou pouco elevada, geralmente inferior a $200 \mathrm{nd} / \mathrm{dL}(3)$.

O objetivo deste relato é apresentar um caso clínico no qual uma dosagem de testosterona total falsamente elevada dificultou o diagnóstico clínico da paciente.

\section{RELATO DO CASO}

Uma paciente do sexo feminino, portadora de diabetes tipo 1 e nefropatia diabética em diálise peritoneal contínua, referiu acne há cerca de 6 meses.

Até há 1 ano seus ciclos menstruais eram regulares e passou, então, a ter metrorragia, por um período de quatro meses, quando foi feito, em outro serviço, o diagnóstico de cisto de ovário. A paciente não se tratou e, há nove meses, encontra-se sem menstruar. Além da acne, não referia qualquer sinal ou sintoma de hiperandrogenismo, como alteração da voz, aumento de massa muscular ou queda de cabelo, alterações mamárias ou de libido. Negava ondas de calor. Nunca engravidou, apesar de ter vida sexual ativa e não utilizar nenhum método de contracepção.

Ao exame físico, apresentava-se em bom estado geral, com índice de massa corporal de $23,6 \mathrm{~kg} / \mathrm{m}^{2} \mathrm{e}$ pressão arterial de 150 x $90 \mathrm{mmHg}$. Acne em face, caracterizada pela presença de pápulas e em menor número pústulas (no total menos de 10), distribuídas em região malar. Distribuição de pelos corporais e cabelos normais. Sua voz era feminina e a massa muscular, compatível com o sexo. Não apresentava acantose nigricante, hematomas ou estrias. As mamas estavam normais, Tanner $\mathrm{V}$, sem massas ou galactorreia. O exame ginecológico não apresentou anormalidades e não havia clitoromegalia.

Quando de sua avaliação inicial, encontrava-se em uso de insulina glargina, lispro, sevelamer, eritropoietina e anlodipina. Todas as amostras de sangue para avaliação de parâmetros bioquímicos e hormonais foram coletadas no período da manhã, entre às 7 horas e 9 horas, após jejum noturno de 8 horas. A avaliação laboratorial pré-diálise demonstrou função hepática normal, creatinina de $11 \mathrm{mg} / \mathrm{dL}$, ureia de $172 \mathrm{mg} /$ $\mathrm{dL}$, hemoglobina de $7,4 \mathrm{~g} / \mathrm{dL}$ e hemoglobina glicada de 7,0\%. O perfil hormonal encontra-se na tabela 1 . A concentração de testosterona total elevada, de 612 $\mathrm{ng} / \mathrm{dL}$ (normal < $90 \mathrm{ng} / \mathrm{dL}$ ), à qual correspondeu uma concentração de testosterona livre, calculada por meio da fórmula de Vermeulen (4), de $255 \mathrm{pM} / \mathrm{L}$ (normal de 20 a $45 \mathrm{pM} / \mathrm{L}$ ), foi confirmada em outra amostra, com uma concentração de $435 \mathrm{ng} / \mathrm{mL}$. Uma terceira amostra de sangue foi coletada, separando-se uma alíquota para dosagem por radioimunoensaio e outra para dosagem por cromatografia líquida de alta performance (HPLC), como método preparativo e espectrometria de massa em tandem para quantificação (5), com resultados de $335 \mathrm{ng} / \mathrm{mL}$ e $21 \mathrm{ng} / \mathrm{mL}$, respectivamente.

Tabela 1. Perfil hormonal da paciente

\begin{tabular}{|c|c|c|c|c|c|c|c|c|}
\hline Hormônio & $\begin{array}{l}\text { LH } \\
\text { (UI/L) }\end{array}$ & $\begin{array}{l}\text { FSH } \\
\text { (UI/L) }\end{array}$ & $\begin{array}{c}E_{2} \\
(p M / L)\end{array}$ & $\begin{array}{c}P \\
(\mathrm{ng} / \mathrm{mL})\end{array}$ & $\begin{array}{l}T \text { total } \\
\text { (ng/dL) }\end{array}$ & $\begin{array}{c}\text { T livre } \\
\text { (pMol/L) }\end{array}$ & $\begin{array}{l}\text { DHEAS } \\
\text { (ng/mL) }\end{array}$ & $\begin{array}{l}\text { SHBG } \\
\text { (nM/L) }\end{array}$ \\
\hline Valor & 3,3 & 5,8 & 67 & 1,9 & 612 & 255 & 2.030 & 51 \\
\hline Método & IMFA & IMFA & EFM & EFM & EFM & Calculada* & EQM & IMFA \\
\hline Normal$^{\star *}$ & $2,2-6,8$ & $2,4-9,3$ & $22-215$ & $0,4-1,1$ & $<90$ & $20-45 \mathrm{pMol} / \mathrm{L}$ & $988-3400$ & $22-130$ \\
\hline
\end{tabular}

LH: hormônio luteinizante; FSH: hormônio folículo-estimulante; $E_{2}$ : estradiol; P: progesterona; T total: testosterona total; T livre: testosterona livre, DHEAS: sulfato de deidroepiandrosterona; SHBG: globulina ligadora dos hormônios esteroides sexuais; IMFA: ensaio imunofluorimétrico; EFM: ensaio fluorimétrico; EQM: ensaio eletroquimioluminescente.

* Calculada por meio da fórmula de Vermeulen (4).

** Para a faixa etária da paciente, na fase folicular do ciclo menstrual. 
Ultrassonografia pélvica por via transvaginal demonstrou útero com volume de $71,3 \mathrm{cc}$, ovário direito com 4,6 cc e ovário esquerdo com 8,6 cc, ambos com cistos de aspecto folicular. Tomografia computadorizada demonstrou adrenais de localização e forma normais.

\section{DISCUSSÃO}

Embora a acne seja considerada usualmente como um sinal de excesso androgênico, não está clara a prevalência de hiperandrogenemia em pacientes com acne, motivo pelo qual esse sinal deve ser valorizado com cautela quando estiver presente isoladamente. No caso em discussão, a presença de doenças crônicas dificulta a avaliação da paciente, pois nessas situações é provável que o distúrbio menstrual seja relacionado à doença de base e independente de hiperandrogenemia. $\mathrm{Na}$ avaliação inicialmente realizada, foi solicitada uma dosagem de testosterona total, cujo resultado demonstrou um valor muito elevado. Uma concentração de testosterona total maior que $200 \mathrm{ng} / \mathrm{dL}$ tem sido utilizada como um marcador para a presença de tumores virilizantes (6). Entretanto, esse número não se originou de um estudo sistemático. Por outro lado, foi demonstrado que o principal indicador para a presença de tumores virilizantes é o quadro clínico (presença dos sintomas e sinais de virilização), e não o quadro laboratorial $(7,8)$. Após investigação apropriada, que resultou negativa, questionou-se nessa paciente o real significado da concentração de testosterona total, uma vez que estavam ausentes outros sinais de manifestação de excesso androgênico. Dentre as possibilidades levantadas para explicar essa dissociação entre o quadro laboratorial e clínico, considerou-se a presença das doenças associadas, insuficiência renal crônica em diálise e diabetes tipo l, e questões de ordem metodológica.

Em pacientes do sexo masculino portadores de insuficiência renal crônica, observa-se, em geral, diminuição da concentração da testosterona total e da testosterona livre em vez de aumento (9-13), com uma concentração elevada de LH e FSH, indicando um comprometimento gonadal primário. Tipicamente essa alteração se observa antes do comprometimento grave da função renal. Essas alterações não são corrigidas pela hemodiálise, que não interfere com a concentração de testosterona total, mas são completamente reversíveis pelo transplante renal (14). De acordo com esses dados e supondo que sejam válidos também para o sexo femi- nino, nem a insuficiência renal nem a diálise poderiam explicar a concentração elevada de testosterona observada nessa paciente.

Alteração menstrual é comum em pacientes com diabetes tipo 1, estando presente em aproximadamente $20 \%$ das pacientes (15-20), incidência essa maior que a observada em mulheres normais, de $8 \%(21)$. Inicialmente tal anormalidade foi atribuída ao mau controle do diabetes (17), mas posteriormente se demonstrou que sua presença não decorria do grau de controle (19). Posteriormente, foram demonstradas anormalidades do eixo gonadotrófico em pacientes com diabetes tipo 1 e distúrbio menstrual quando comparadas a pacientes sem distúrbio menstrual (20). Essa maior prevalência de distúrbio menstrual se acompanha também de uma maior incidência de hiperandrogenismo, encontrado em aproximadamente $40 \%$ das pacientes, representado principalmente pelo hirsutismo $(16,22)$, porém com um grau menor quando comparado a mulheres hiperandrogênicas não diabéticas. Por outro lado, a incidência de acne e alopecia androgênica não foi diferente da encontrada na população geral $(16,22)$.

Quanto à concentração dos andrógenos, pacientes diabéticas com hiperandrogenismo apresentam uma concentração maior de testosterona total e livre e androstenediona quando comparadas a mulheres normais, enquanto as pacientes diabéticas sem hiperandrogenismo não apresentam alteração significativa desses hormônios (16). O resultado de estudo com testes dinâmicos é compatível com uma origem ovariana pura da hiperandrogenemia (22). Entretanto, a concentração máxima de testosterona total observada nas pacientes hiperandrogênicas diabéticas foi inferior a $110 \mathrm{ng} / \mathrm{dL}$, concentração essa muito menor que a observada neste caso.

À semelhança da hiperandrogenemia, observa-se também uma maior incidência de ovários com morfologia policística em pacientes diabéticas tipo 1, mesmo quando se empregam critérios estritos para esse diagnóstico (17), da ordem de 50\%, contra $13 \%$ da incidência no grupo controle (16). De acordo com esses dados, dois estudos encontraram uma maior incidência da síndrome dos ovários policísticos em diabéticas tipo l: uma incidência de $40,5 \%$ foi observada em mulheres espanholas com diabetes tipo 1 , contra $6,5 \%$ da observada em mulheres normais (16), enquanto no Chile foi observada uma incidência variando de acordo com o critério diagnóstico empregado (23), de $12 \%$ com o critério do National Institute of Health (24), 31\% com o 
critério da The Androgen Excess and PCOS Society (25) e $40,5 \%$ com o critério do Consenso de Rotterdam (26).

No presente caso, as concentrações de testosterona total e livre foram elevadas, enquanto a concentração de DHEAS foi normal. Devemos enfatizar que essa concentração de testosterona é muito superior à habitualmente encontrada nas síndromes hiperandrogênicas não virilizantes, mesmo em diabéticas tipo $1 \mathrm{com}$ síndrome dos ovários policísticos. Esses dados excluem a síndrome dos ovários policísticos como diagnóstico. Da mesma maneira, o estudo morfológico das adrenais, por meio da tomografia computadorizada, e o do ovário, por meio do ultrassom, resultaram normais. Assim, na ausência de sinais de virilização e de imagem compatível, foram excluídas as neoplasias funcionantes de adrenal ou de ovário.

A dosagem de testosterona total foi introduzida na prática laboratorial no início da década de 1970, por meio do radioimunoensaio (27). Na metodologia inicial, havia a necessidade do preparo da amostra a ser dosada (fase pré-analítica), para, em seguida, proceder-se à dosagem da testosterona, pela incubação por várias horas com material radioativo (testosterona triciada), seguida de uma fase de separação com emprego de carvão ativado. Posteriormente, vários avanços, como a produção de anticorpos de maior afinidade, utilização de marcadores não radioativos e eliminação da fase pré-analítica, permitiram o emprego da dosagem de testosterona total na rotina da prática ambulatorial. Entretanto, esses avanços se acompanharam de uma diminuição da especificidade dos métodos, principalmente na faixa de concentração de testosterona baixa, como a encontrada em crianças e mulheres normais $(28,29)$. Por outro lado, concentração falsamente elevada pode ser encontrada devido à presença de interferentes na dosagem (29).

Para a avaliação da testosterona livre, recomenda-se o método de diálise de equilíbrio (29), que é impraticável para a rotina laboratorial. Como opções a esse método, são usualmente utilizados a dosagem da testosterona não ligada à SHBG, por meio da técnica da precipitação com sulfato de amônio (30), ou o cálculo por meio das concentrações da testosterona total e da SHBG, realizado pela fórmula de Vermeulen (4). A obtenção da testosterona livre por esses métodos está na dependência da dosagem da testosterona total.

Considerando-se a possibilidade de interferentes nas dosagens de testosterona total e androstenediona, no presente caso, optou-se por uma dosagem por meio da
HPLC como método preparativo e espectrometria de massa em tandem para quantificação. Trata-se de técnica com alta especificidade e sensibilidade, sendo considerada referência para a dosagem de hormônios esteroides (31). No caso da testosterona, várias publicações fundamentadas em HPLC como método preparativo e espectrometria de massa em tandem para quantificação foram recentemente apresentadas e demonstraram sua superioridade em relação aos métodos tradicionais, especialmente em amostras de mulheres e crianças (32-34).

É possível que a presença de acne nessa paciente, uma vez excluídas as causas mais frequentes, possa ser atribuída a uma complicação da diálise, descrita tanto em pacientes em hemodiálise $(35,36)$ quanto em pacientes em diálise peritoneal (37), por mecanismo desconhecido. O tratamento instituído nesses casos é convencional, até mesmo quanto ao uso de isotretionina.

Em conclusão, apresentamos o caso de uma paciente cuja dosagem de testosterona elevada conduziu à pesquisa de uma síndrome virilizante, de etiologia neoplásica. Entretanto, a dissociação entre o resultado laboratorial e clínico levou a considerar outras hipóteses, entre as quais interferência no método de dosagem. O emprego de HPLC como método preparativo e espectrometria de massa para a dosagem da testosterona total permitiu concluir tratar-se de um caso de concentração falsamente elevada.

Agradecimentos: ao Laboratório Fleury de São Paulo, pela dosagem de testosterona por cromatografia líquida.

Declaração: os autores declaram não haver conflitos de interesse científico neste estudo.

\section{REFERÊNCIAS}

1. Cibula D, Hill M, Starka L. The best correlation of the new index of hyperandrogenism with the grade of increased hair. Eur J Endocrinol. 2000;143:405-8.

2. Kirschner MA, Bardin CW. Androgen production and metabolism in normal and virilized women. Metabolism. 1972;21:667-88.

3. Azziz R, Sanchez LA, Knochenhauer ES, Moran C, Lazenby J, Stephens KC, et al. Androgen excess in women: experience with over 1000 consecutive patients. J Clin Endocrinol Metab. 2004;89:453-62.

4. Vermeulen A, Verdonck L, Kaufman JM. A critical evaluation of simple methods for estimation of free testosterone in serum. J Clin Endocrinol Metab. 1999;84:3666-72.

5. Wang C, Catlin DH, Demers LM, Starcevic B, Swerdloff RS. Measurement of total serum testosterone in adult men: comparison of current laboratory methods versus liquid chromatography-tadem mass spectrometry. J Clin Endocrinol Metab. 2004;89:534-43.

6. Meldrum DR, Abraham GE. Peripheral and ovarian venous concentrations of various steroid hormones in virilizing ovarian tumors. Obstet Gynecol. 1979;53:36-43. 
7. Surrey ES, de Ziegler D, Gambone JC, Judd HL. Preoperative localization of androgen-secreting tumors: clinical, endocrinologic, and radiologic evaluation of ten patients. Am J Obstet Gynecol. 1988;158:1313-22.

8. Derksen J, Nagesser SK, Meinders AE, Haak HR, van de Velde CJ. Identification of virilizing adrenal tumors in hirsute women. $\mathrm{N}$ Engl J Med. 1994;331:968-73.

9. Guevara A, Bidt D, Hallberg M, Zorn E, Pholman C, Wieland R. Serum gonadotropin and testosterone levels in uremic males undergoing intermittent dialysis. Metabolism. 1969;18:1062-6.

10. Stewart-Bentley M, Gans D, Horton R. Regulation of gonadal function in uremia. Metabolism. 1974;23:1065-72.

11. Krolner B. Serum levels of testosterone and luteinizing hormone in patients with chronic renal disease. Acta Med Scand. 179;205:623-7.

12. Schmidt A, Luger A, Horl W. Sexual hormone abnormalities in male patients with renal failure. Nephrol Dial Transplant. 2002;17:368-71.

13. Holdsworth S, Atkins RC, de Kretser DM. The pituitary-testicular axis in men with chronic renal failure. $\mathrm{N}$ Engl $\mathrm{J}$ Med. 1977;296:1245-9.

14. Lim VS, Fang VS. Gonadal dysfunction in uremic men. A study of the hypothalamo-pituitary-testicular axis before and after renal transplantation. Am J Med. 975;58:655-62.

15. Escobar-Morreale HF, Roldan B, Barrio R, Alonso M, Sancho J, de la Calle $\mathrm{H}$, et al. High prevalence of the polycystic ovary syndrome and hirsutism in women with type 1 diabetes mellitus. J Clin Endocrinol Metab. 2000;85:4182-7.

16. Codner E, Soto N, Lopez P, Trejo L, Avila A, Eyzaguirre FC, et al. Diagnostic criteria for polycystic ovary syndrome and ovarian morphology in women with type 1 diabetes mellitus. J Clin Endocrinol Metab. 2006;91:2250-6.

17. Schroeder B, Hertweck SP, Sanfilippo JS, Foster MB. Correlation between glycemic control and menstruation in diabetic adolescents. J Reprod Med. 2000;45:1-5.

18. Strotmeyer ES, Steenkiste AR, Foley JrTP, Berga SL, Dorman JS. Menstrual cycle differences between women with type 1 diabetes and women without diabetes. Diabetes Care. 2003;26:1016-21.

19. Djursing H, Hagen C, Andersen A, Svenstrup B, Bennett P, Mølsted Pedersen L. Serum sex hormone concentrations in insulin dependent diabetic women with and without amenorrhoea. Clin Endocrinol. 1985;23:147-54.

20. Virdis R, Zampolli M, Street ME, Vanelli M, Potau N, Terzi C, et al. Ovarian $17 \alpha$-hydroxyprogesterone responses to $\mathrm{GnRH}$ analog testing in oligomenorrheic insulin-dependent diabetic adolescents. Eur J Endocrinol. 1997;136:624-9.

21. Vink JM, Sadrzadeh S, Lambalk CB, Boomsma DI. Heritability of polycystic ovary syndrome in a Dutch twin-family study. J Clin Endocrinol Metab. 2006;91:2100-4.

22. Roldán $B$, Escobar-Morreale HF, Barrio R, de La Calle $H$, Alonso $M$, García-Robles $R$, et al. Identification of the source of andro- gen excess in hyperandrogenic type 1 diabetic patients. Diabetes Care. 2001;24:1297-9.

23. Codner E, Escobar-Morreale HF. Clinical review: hyperandrogenism and polycystic ovary syndrome in women with type 1 diabetes mellitus. J Clin Endocrinol Metab. 2007;92:1209-16.

24. Zawadeski JK, Dunaif A. Diagnostic criteria for PCOS: towards a more rational approach. In: Dunaif A, Givens JR, Haseltine FP, Merriam GR, eds. PCOS. Boston: Blackwell Scientific; 1992. p. 377-84.

25. Azziz R, Carmina E, Dewailly D, Diamanti-Kandarakis E, Escobar-Morreale HF, Futterweit W, et al. Positions statement: criteria for defining polycystic ovary syndrome as a predominantly hyperandrogenic syndrome: an Androgen Excess Society guideline. J Clin Endocrinol Metab. 2006;91:4237-45.

26. Rotterdam ESHRE/ASRM - Sponsored PCOS Consensus Workshop Group. Revised 2003 consensus on diagnostic criteria and long-term health risks related to polycystic ovary syndrome. Fertil Steril. 2004;81:19-31.

27. Furuyama $S$, Mayes DM, Nugent CA. A radioimmunoassay for plasma testosterone. Steroids. 1970;16:415-28.

28. Herold DA, Fitgerald RL. Immunoassays for testosterone in women: better than a guess? Clin Chem. 2003;49:1250-1.

29. Rosner W. Errors in the measurement of plasma free testosterone. J Clin Endocrinol Metab. 1997;82:2014-5.

30. Tremblay RR, Dube JY. Plasma concentration of free and non-TeBG bound testosterone in women on oral contraceptives. Contraception. 1974;10:599-605.

31. Vieira JGH, Nakamura OH, Carvalho VM. Dosagem de cortisol e cortisona livres urinários empregando cromatografia líquida associada a espectrometria de massa em tandem (LC-MS/MS). Arq Bras Endocrinol Metab. 2005;49:291-8.

32. Wang C, Catlin DH, Demers LM, Starcevic B, Swerdloff RS. Measurement of total serum testosterone in adult men: comparison of current laboratory methods versus liquid chromatography-tadem mass spectrometry. J Clin Endocrinol Metab. 2004;89:534-43.

33. Cawood ML, Field HP, Ford CG, Gillingwater S, Kicman A, Cowan $D$, et al. Testosterone measurement by isotope-dilution liquid chromatography-tandem mass spectrometry: validation of a method for routine clinical practice. Clin Chem. 2005;51:1472-9.

34. Kushnir MM, Rockwood AL, Roberts WL, Pattison EG, Bunker $A M$, Fitzgerald RL, et al. Performance characteristics of a novel tandem mass spectrometry assay for serum testosterone. Clin Chem. 2006;52:120-8.

35. Grange F, Mitschler A, Genestier S, Guillaume JC. Severe pruriginous acne in dialysed renal failure. Diagnostic difficulties and efficacy of isotretinoin. Ann Dermatol Venereol. 2001;128:1215-9.

36. Fuchs E, Lynfield Y. Dialysis acne. J Am Acad Dermatol. 1990;23(1):125-9.

37. Lin J, Shih I, Yu C. Hemodialysis-related nodulocystic acne treated with isotretinoin. Nephron. 1999;81:146-50. 